Supporting Information for

\title{
Influence of Surface Ligand Molecular Structure on Phospholipid Membrane Disruption by Cationic Nanoparticles
}

Yongqian Zhang ${ }^{\dagger \uparrow}$, Udaya Dahal ${ }^{\ddagger}$, Z. Vivian Feng", Zeev Rosenzweig ${ }^{\perp}$, Qiang Cui, Robert J. Hamers $^{\dagger *}$

${ }^{\dagger}$ University of Wisconsin-Madison, Department of Chemistry, Madison, WI, USA, 53706

¥Boston University, Department of Chemistry, Boston, MA, USA, 02215

${ }^{\perp}$ University of Maryland Baltimore County, Department of Chemistry, Baltimore, MD, USA, 21250

"Augsburg University, Chemistry Department, Minneapolis, MN, USA, 55454

*Corresponding author

S1. Surface characterization of cationic functionalized diamond NPs ....................... page 2

S2. Fluorescence-based lysis assay plot versus diamond NP concentration ............... page 4

S3. ${ }^{1} \mathrm{H}-T_{2}$ NMR analysis of PAH-DNPs. ............................................................ Page 5

S4. 1D- ${ }^{31}$ P-NMR spectra of POPC, POPG lipids and POPC: POPG SUV ................. page 7

S5. MD simulation of diffusion coefficients of lipids in SUVs .................................. page 8

S6. MD simulation of the effect of ligand density .................................................... page 9

S7. MD simulation of the effect of coarse-grained bead type for the DNP core.......... page 11 
S1. Surface characterization of cationic functionalized diamond NPs. DNPs functionalized in an identical manner with these same surface ligands were used previously. Complete characterization data was published in that earlier work and is therefore not repeasted here.
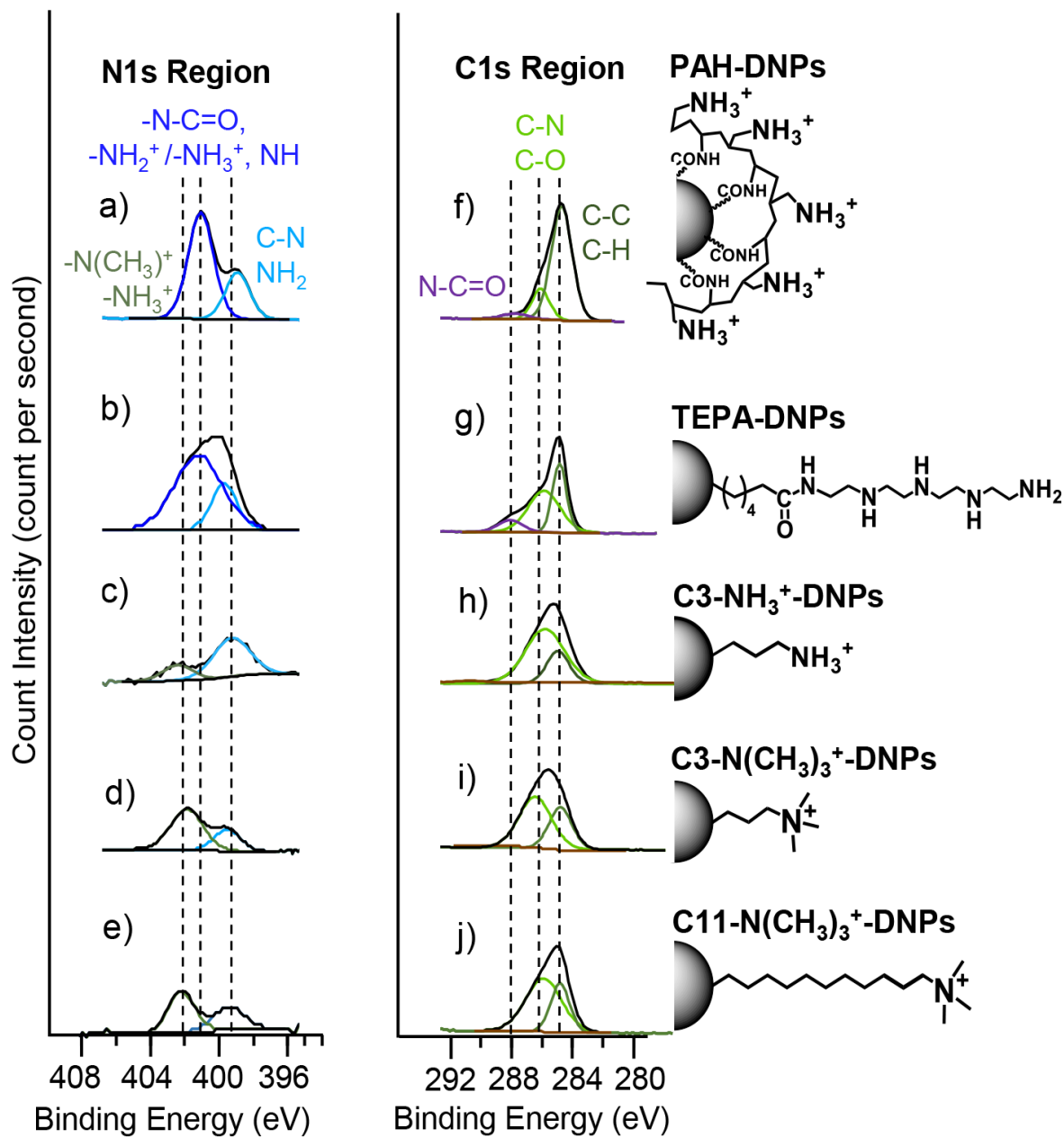

Fig S1. XPS spectra of N(1s) regions (a-e) and C(1s) regions (f-j) of PAH-DNPs, TEPADNPs, C3-NH3+-DNPs, $\mathrm{C} 3-\mathrm{N}(\mathrm{CH} 3)_{3}{ }^{+}$-DNPs and $\mathrm{C} 11-\mathrm{N}\left(\mathrm{CH}_{3}\right)_{3}{ }^{+}$-DNPs. 


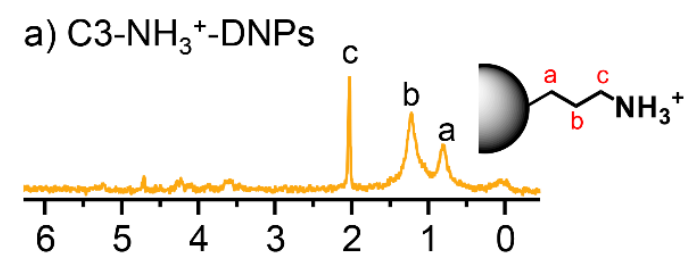

b) $\mathrm{C} 3-\mathrm{N}\left(\mathrm{CH}_{3}\right)_{3}{ }^{+}$-DNPs
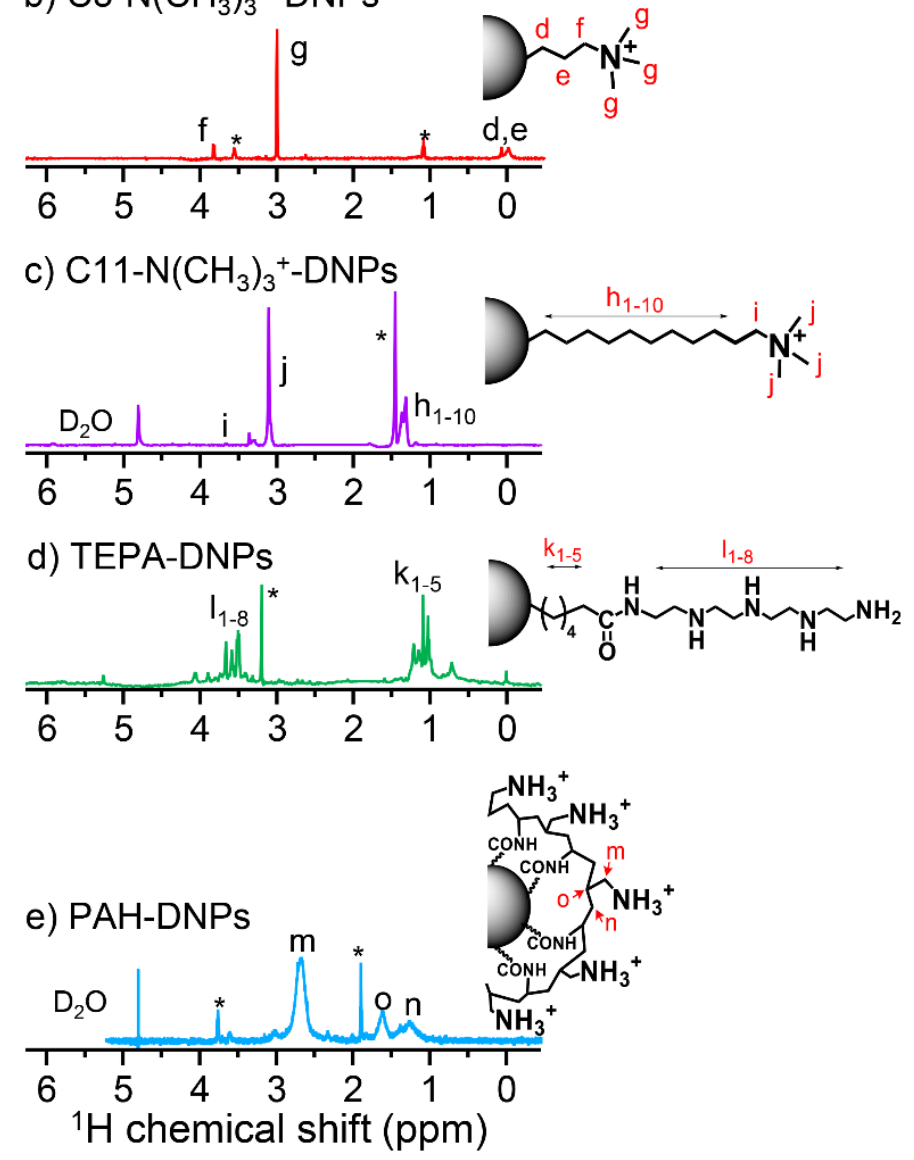

Fig S2. ${ }^{1} \mathrm{H}$ NMR spectra of a) $\mathrm{C} 3-\mathrm{NH}_{3}{ }^{+}$-DNPs, b) $\mathrm{C} 3-\mathrm{N}\left(\mathrm{CH}_{3}\right)_{3}{ }^{+}$-DNPs c) C11$\mathrm{N}\left(\mathrm{CH}_{3}\right)_{3}{ }^{+}$-DNPs, d) TEPA-DNPs and e) PAH-DNPs. Asterisk denotes solvent peaks. 
S2. Fluorescence-based lysis assay plot versus diamond NP concentration. The DNP particle concentration was determined by gravimetric method.

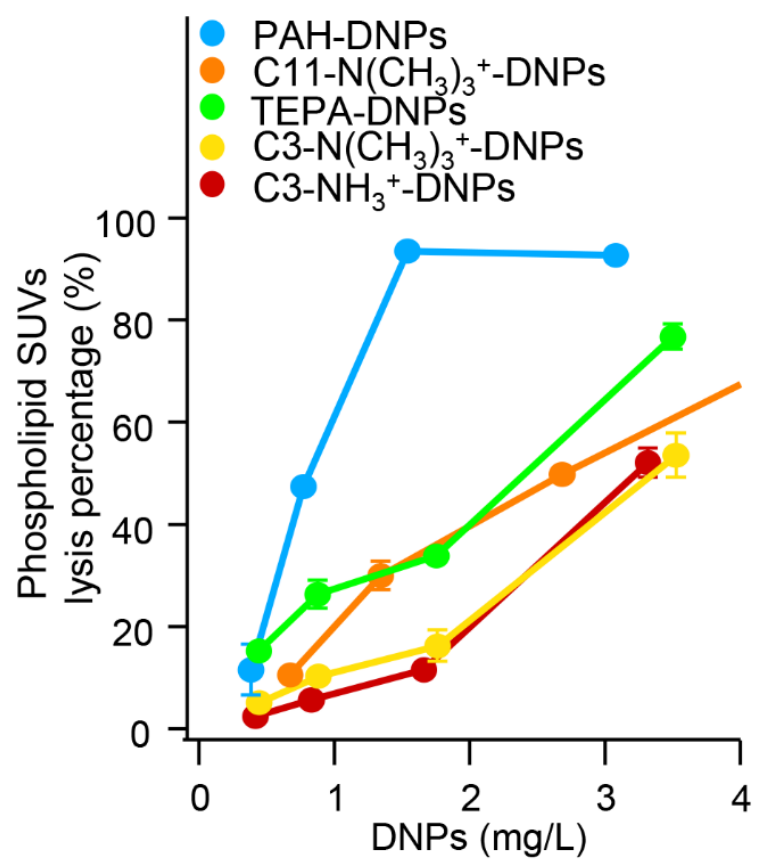

Fig. S3. Fluorescence-based lysis assay measurements versus the DNPs concentrations. 


\section{S3. ${ }^{1} \mathrm{H}-\mathrm{T}_{2}$ NMR analysis of PAH-DNPs.}

The PAH-DNPs were functionalized using a radical-based functionalization method. The $T_{2}$ values that are shown in Table S1 result from the average of three PAH peaks.

Table S1. ${ }^{3} \mathrm{H}-T_{2}$ relaxation results of all three $\mathrm{PAH}$ peaks.

\begin{tabular}{c|cccc}
\hline \hline $\begin{array}{c}{ }^{1} \mathrm{H} \text { chemical shift } \\
(\mathrm{ppm})\end{array}$ & \multicolumn{2}{c}{$\begin{array}{c}\text { Dynamic region 1 } \\
\text { (more rigid motion) }\end{array}$} & \multicolumn{2}{c}{$\begin{array}{c}\text { Dynamic region 2 } \\
\text { (more flexible motion) }\end{array}$} \\
\hline & $\begin{array}{c}T_{2} \\
(\mathrm{~ms})\end{array}$ & $\begin{array}{c}\text { population } \\
(\%)\end{array}$ & $\begin{array}{c}T_{2} \\
(\mathrm{~ms})\end{array}$ & $\begin{array}{c}\text { population } \\
(\%)\end{array}$ \\
\hline 1.3 & 5.6 & 73.7 & 20 & 26.3 \\
1.6 & 5.4 & 81.2 & 33.3 & 18.8 \\
2.7 & 2.3 & 69.8 & 12.5 & 30.2 \\
& & & & \\
Average & $4.4 \pm 1.5$ & $74.9 \pm 4.7$ & $21.9 \pm 8.6$ & $25.1 \pm 4.7$ \\
\hline \hline
\end{tabular}


a)
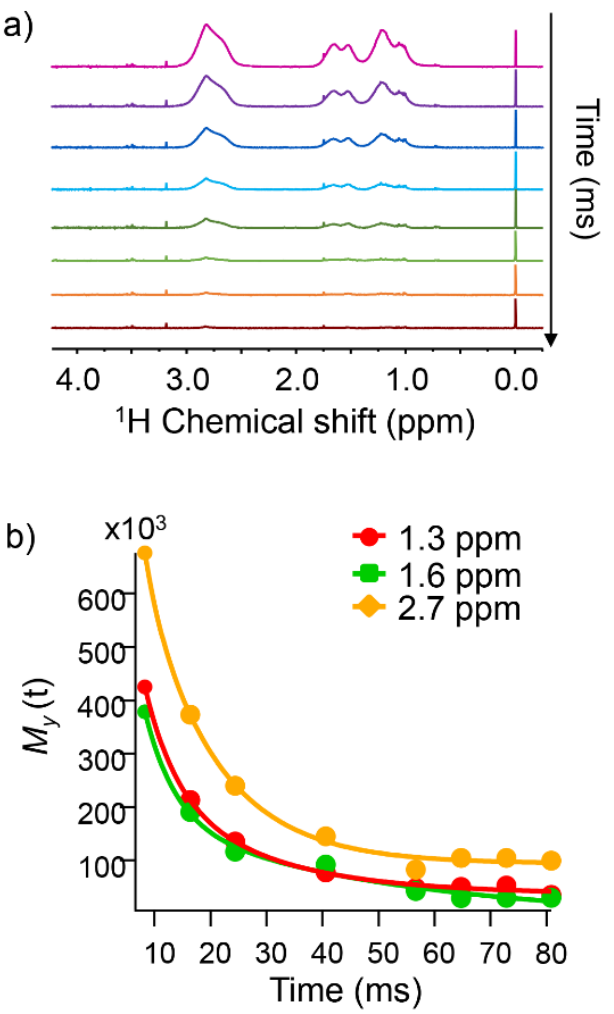

Fig. S4. a) A series of ${ }^{1} \mathrm{H}$ spectra result from the CPMG experiment with increasing times and b) $T_{2}$ exponential decay and associated curve-fits of all three PAH peaks versus time. 
S4. 1D- ${ }^{31}$ P-NMR spectra of POPC, POPG lipids (not packing into a SUV) and POPC: POPG SUV.

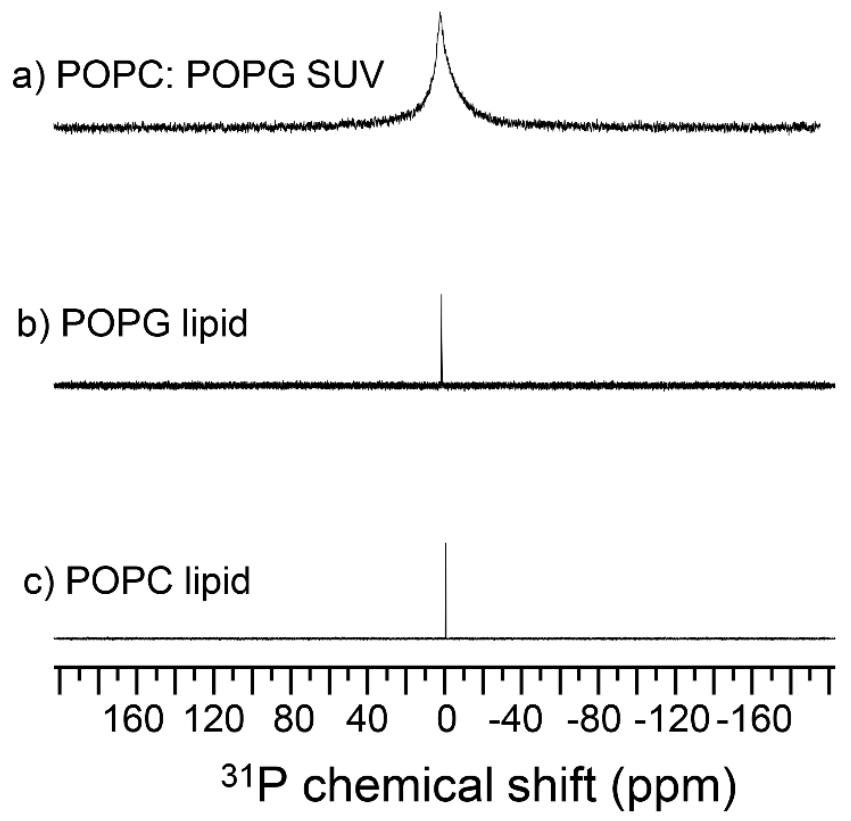

Fig. S5. ${ }^{31}$ P NMR spectra of a) POPC: POPG SUV, b) POPG lipid without packing into a SUV and c) POPC lipid without packing into a SUV. 


\section{S5. MD simulation of diffusion coefficients of the lipids in SUVs}

The diffusion constants of the phospholipids from inner and outer layers computed from the coarse-grained simulations are shown below in Table S.2. In those calculations, since the diffusion of lipids in a vesicle occurs along a curved spherical surface, we consider the angular mobility of the lipids: equation (S1) is used to calculate mean square displacement of the lipids in the vesicle (Apaza, Leonardo, and Mario Sandoval. "Brownian self-driven particles on the surface of a sphere." Physical Review E 96.2 (2017): 022606),

$$
\left\langle(\theta(t)-\theta(0))^{2}\right\rangle=2 \frac{D}{r^{2}} t
$$

where $\theta$ is the angle between the position vector $(\vec{r})$ of the lipid head group and the local surface normal, as shown in Figure S6. As shown in Table S2, the diffusion constants for lipids in the two leaflets can differ substantially, almost by a factor two, which is qualitatively consistent with the $T_{2}$ relaxation results.

Table S2. Calculated diffusion coefficients $(D)$ of PC and PG lipids in the inner and outer layers of SUV without DNPs by

MD simulations.

\begin{tabular}{|c|c|}
\hline Species & $\begin{array}{c}\begin{array}{c}\text { Diffusion coefficients } \\
\left(\mathrm{cm}^{2} \cdot \mathrm{s}^{-1}\right)\end{array} \\
\end{array}$ \\
\hline inner PC lipids & $1.32 \mathrm{E}-07$ \\
\hline inner PG lipids & $1.67 \mathrm{E}-07$ \\
\hline outer PC lipids & $2.02 \mathrm{E}-07$ \\
\hline outer PG lipids & $2.36 \mathrm{E}-07$ \\
\hline
\end{tabular}




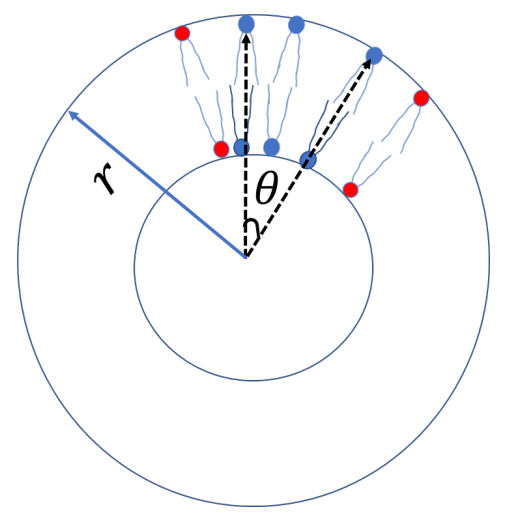

Fig. S6. Schematic illustration for the calculation of lipid diffusion in a PC-PG SUV.

\section{S6. MD simulation of the effect of ligand density}

To probe the effect of ligand density, we compare results with DNP functionalized with 8 and 16 $\mathrm{PAH}_{20}$ chains on the surface. The results in Fig. S7 show that higher ligand density does not necessarily lead to greater interactions and deeper membrane penetration by the DNP. For example, in DNP with $8 \mathrm{PAH}_{20}$ chains, each individual PAH chain is extended, which allows the DNP to interact strongly with the SUV and induce significant membrane deformations. When the ligand density increases to 16 , the PAH chains tend to wrap around the DNP and do not exhibit extended conformations; this reduces interactions with the SUV membranes. The radial distribution function of phosphate relative to the center of mass of the SUV shows the same trend: the phosphate distribution is perturbed more significantly by the DNAP with $8 \mathrm{PAH}_{20}$ chains than with $16 \mathrm{PAH}_{20}$ chains. This result further demonstrates that the conformational of surface ligand plays a key role in modulating NP-membrane interactions. 
a)

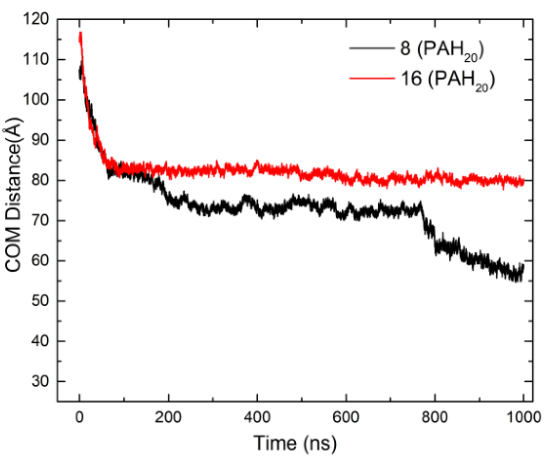

c)

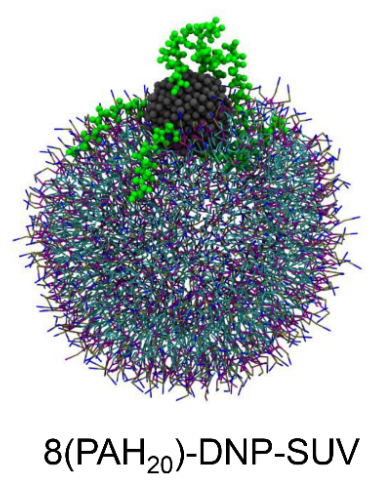

b)

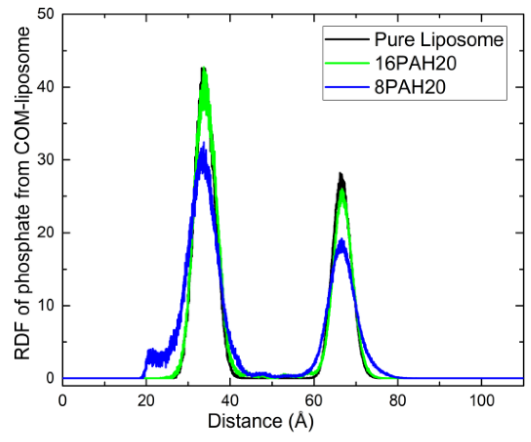

d)

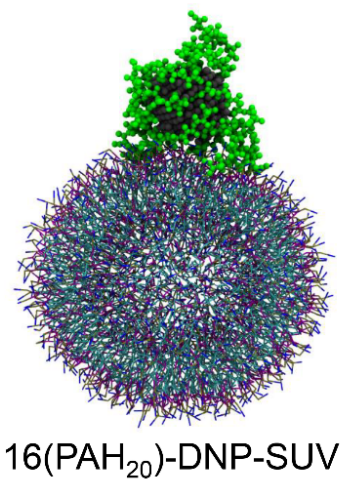

Fig. S7. a) Distance from center of mass (COM) between the PAH-DNPs and the SUV obtained from MD simulations. b) Radial distribution function of lipid phosphate groups with respect to the COM of the SUV, for pure SUV, $16 \mathrm{PAH}_{20}-\mathrm{DNP}$ and $8 \mathrm{PAH}_{20}$-DNP. Snapshots of the PAH-DNP-SUV complex with $8 \mathrm{PAHP}_{20}$ and $16 \mathrm{PAH}_{20}$ are shown in panel c and d, respectively. 


\section{S7. MD simulation of the effect of the coarse-grained (GC) bead type for the DNP core}

To probe the effect of the DNP core on the DNP-membrane interaction, we conducted additional simulation in which the DNP core beads are changed to type P1, which is more hydrophobic than P4 (used in the main text and Fig. S7). We use $16 \mathrm{PAH}_{20}-\mathrm{DNP}$ as an example. As shown in Fig. S8, the COM separation between the DNP and SUV is generally similar to that observed with simulations that employed the P4 beads for the DNP core (Fig. S7): after about 1 microsecond, the COM separation reaches between 80 and $90 \AA$ for both simulations. Therefore, the impact of the DNP core on the DNP-SUV interaction is relatively minor.

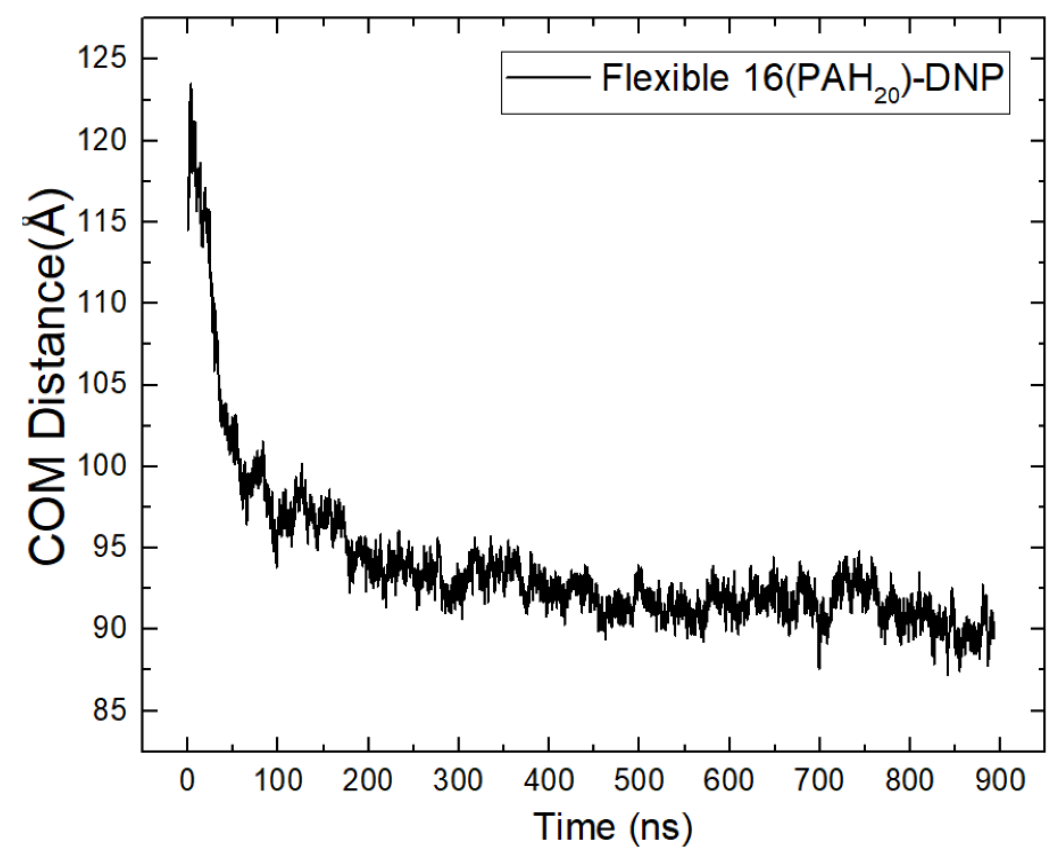

Fig. S8. Time evolution of the center of mass distances between SUV and $16 \mathrm{PAH}_{20^{-}}$ DNP. 